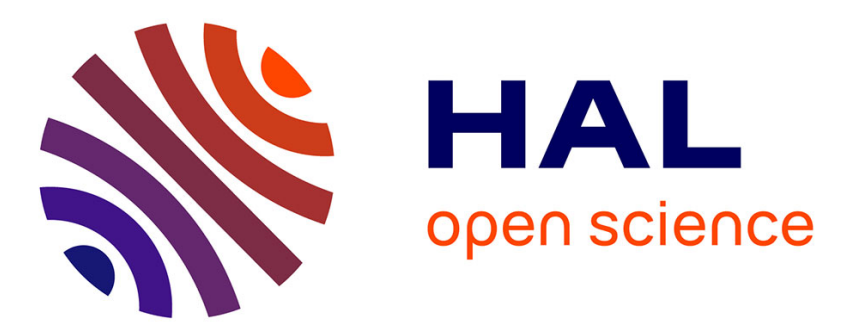

\title{
Shape Programming by Modulating Actuation over Hierarchical Length Scales
}

Tian Gao, Emmanuel B Siéfert, Antonio Desimone, Benoît Roman

\section{To cite this version:}

Tian Gao, Emmanuel B Siéfert, Antonio Desimone, Benoît Roman. Shape Programming by Modulating Actuation over Hierarchical Length Scales. Advanced Materials, 2020, 32 (7), 10.1002/adma.202004515 . hal-03442616

\section{HAL Id: hal-03442616 https://hal.science/hal-03442616}

Submitted on 23 Nov 2021

HAL is a multi-disciplinary open access archive for the deposit and dissemination of scientific research documents, whether they are published or not. The documents may come from teaching and research institutions in France or abroad, or from public or private research centers.
L'archive ouverte pluridisciplinaire HAL, est destinée au dépôt et à la diffusion de documents scientifiques de niveau recherche, publiés ou non, émanant des établissements d'enseignement et de recherche français ou étrangers, des laboratoires publics ou privés. 


\title{
Shape Programming by Modulating Actuation over Hierar- chical Length Scales
}

\section{Tian Gao Emmanuel Siéfert Antonio DeSimone Benô̂t Roman*}

\author{
T. Gao, Dr. E. Siéfert, Prof. B. Roman
}

$\mathrm{PMMH}$

CNRS, ESPCI Paris, Université PSL, Sorbonne Université, Université de Paris

F-75005,Paris, France

E-mail: benoit.roman@espci.fr

Prof. A. DeSimone

MathLab

SISSA-International School for Advanced Studies

Trieste 34136, Italy

Prof. A. DeSimone

The BioRobotics Institute and Department of Excellence in Robotics and AI

Scuola Superiore Sant'Anna

Pisa 56127, Italy

Keywords: Responsive materials, Homogenization, Shape programming, Surface patterning, Actuation

Many active materials used in shape-morphing respond to an external stimulus by stretching or contracting along a director field. The programming of such actuators remains complex because of the single degree of freedom (the orientation) in local actuation. Here, texturing this field in zigzag patterns is shown to provide an extended family of biaxial active stretches out of an otherwise single uniaxial active deformation, opening a larger parameter space. By further modulating the zigzag patterns at the larger scale of the structure, its deployed shape can be controlled. This notion of texturing over hierarchical length-scales follows geometrical principles, and is robust against changes in size and materials. We demonstrate the robustness of our approach by considering three different responsive materials: inextensible flat fabrics, channel bearing elastomer (respectively contracting and expanding perpendicularly to the director field when actuated pneumatically) and 3D-printed thermoplastic (composed of extruded filaments that contract when heated). We show that large-scale shapemorphing structures can be generated and that their geometry can be controlled with high accuracy.

A large family of responsive materials (that deform when a stimulus is imposed) have been developed in recent years as candidates for "materials as machines", because they are capable of directly using available energy to perform a defined function, with applications envisioned in soft and small scale robotics. ${ }^{[1-3]}$ In many implementations, a gradient of actuation through the thickness of a plate provides its spontaneous bending along a single direction. ${ }^{[4,5]}$ But recent years have shown 
that more complex actuation geometries may be achieved using "Gaussian morphing", i.e. the spatial programming of in-plane deformations, or metric distortion, inducing a change in Gaussian curvature. ${ }^{[6-9]}$ In many systems, the deformation is anisotropic and has a uniform magnitude, but can be oriented along a director field. This is the case of Liquid Crystal Elastomers (LCE), ${ }^{[10-15]}$ pneumatic elastomers, ${ }^{[16]}$ the biological sliding of Euglena, ${ }^{[17]}$ photo-responsive materials, ${ }^{[18,}$ 19] swelling restricted by oriented fibers, ${ }^{[20-22]}$ or $3 \mathrm{D}$ printed thermo-responsive filaments. ${ }^{[23,24]}$ Several approaches were used to determine the director field that will generate a target three-dimensional shape, ${ }^{[13,25]}$ but this inverse problem is difficult and there is no guarantee that a solution exists in general. Using a larger family of metrics could ease this inverse problem. In some specific cases, a convenient additional degree of freedom indeed leads to an explicit inverse solution. ${ }^{[16,26]}$ Here we use short wavelength modulation into zigzag patterns to expand the set of available coarse-grained metric changes. We then modulate the geometry of the zigzags at the long wave lengths of the metric gradients required to produce the target Gaussian curvature and the desired shape (Figure 1).

Homogenization over oscillations at short wavelengths is a general and broadly studied concept in mechanics. The cooperative evolution of ferromagnetic domains, ${ }^{[27,28]}$ martensitic microstructures, ${ }^{[29-31]}$ and stripe domains in liquid crystal elastomers ${ }^{[12,32]}$ provide, among others, examples where the macroscopic response of a material (magnetization curves, stress-strain curves) averages at the sample scale the response of microstructures to applied magnetic fields and loads. While the concept of macroscopic response emerging from the cooperative evolution of microstructures has been used extensively in materials science, its application to shape programming problems seems new.

In this article we show that it is possible to significantly extend the family of effective actuation by homogenizing textures made of a piece-wise uniform director field. As highlighted in Figure 
2a, the region of reachable strains goes from typically a line (e.g. changing the temperature for LCEs is restricted to the curve $\lambda_{\perp}=\lambda_{\|}^{-0.5}$, as the volume $\lambda_{\perp}^{2} \lambda_{\|}=1$ is conserved) to an area with hierarchical texturing. This concept applies to all responsive materials that are not conformal (i.e. which do not deform isotropically). As an experimental demonstration, we use three different responsive materials, namely inflatable fabrics, ${ }^{[33]}$ pneumatic "baromorph" elastomers, ${ }^{[16]}$ and standard 3D printed PolyLactic Acid (PLA) filaments. ${ }^{[34]}$ Inflatable fabrics are composed of two flat superimposed sheets sealed together along an elongated pattern of seams, defining locally parallel channels. Upon inflation (Figure 2b, left), the channels acquire a circular cross section while the envelope barely stretches, inducing an in-plane average contraction perpendicular to the seam lines $\lambda_{\perp}<1$ and no contraction along the seam lines $\left(\lambda_{\|}=1\right)$. In baromorph elastomers, aligned airways are embedded in the elastomeric plate. ${ }^{[16]}$ An applied inner pressure (Figure 2b, center) leads to material stretching and an averaged pressure dependent expansion perpendicular to the channels of magnitude $\lambda_{\perp} \geq 1$, while maintaining almost $\lambda_{\|}=1$ along the airways (see experimental section). In 3D-printed PLA structures, the long polymeric chains are aligned and straighten in the nozzle as the melted plastic experiences an extensional flow. ${ }^{[24,34]}$ When the extruded filament is thin enough, it cools down and solidifies before the polymer chains can rearrange, freezing an entropically unfavourable configuration. When heated above the glass transition temperature (but below the melting temperature), the chains regain their isotropic shape. This shape memory effect leads to a macroscopic contraction $\left(\lambda_{\|}<1\right)$ of the material along the printing direction (Figure 2b, right, see experimental section and Supporting Information for more details). $[35,36]$

We consider a general case where in the local cartesian frame $\left(\mathbf{e}_{\|}, \mathbf{e}_{\perp}\right)$, the local metric tensor 
describing the activation stretches can be defined as:

$$
\bar{g}=\left(\begin{array}{cc}
\lambda_{\|}^{2} & 0 \\
0 & \lambda_{\perp}^{2}
\end{array}\right)
$$

In other words, at the "microscale" in Figure 1, the material undergoes an active stretch $\lambda_{\|}$(not necessarily equal to 1) along the director field $\mathbf{e}_{\|}$, and $\lambda_{\perp}$ in the perpendicular direction. The ratio of area change $\lambda_{\Sigma}=\lambda_{\perp} \lambda_{\|}$will play the role of an important invariant in this study.

Consider now the interface between two areas with uniform (but different) director fields $\boldsymbol{e}_{\alpha}$ and $\boldsymbol{e}_{\beta}$ (as shown in Figure 1). The arrangement is kinematically compatible (i.e., the interface is stretched identically by the two adjacent patches) if and only if the boundary line is one of the two bisectrices of directions $\boldsymbol{e}_{\alpha}$ and $\boldsymbol{e}_{\beta}$ (see the Supporting Information). We define $\theta_{0}$ the common angle between the director fields and the interface line. Several patches may be combined into closed loop of the vector field, leading to localized singular Gaussian curvature. ${ }^{[26,37]}$ We are rather interested in continuous metric programming and consider parallel stripes with alternating director field, i.e. a zigzag pattern. Our approach will be to consider periodic zigzag patterns, and evaluate the deformation field that they generate when averaged over a larger length scale (the "mesoscale" in Figure 1).

We first investigate a symmetric periodic pattern where the bands have all the same width. Two consecutive stripes then provide the unit cell of our periodic construction, from which the average active strain may be deduced (as in Figure 3a). By symmetry, the kink line and its perpendicular are the principal direction of the target average stretch, and we find the contraction ratio parallel $L / L_{0}=\Lambda_{1}$ and perpendicular $H / H_{0}=\Lambda_{2}$ to the bisectrix as (see Figure 3a for the definition of the geometrical parameters and Supporting information for details on the derivation):

$$
\Lambda_{1}^{2}=\lambda_{\|}^{2} \cos ^{2} \theta_{0}+\lambda_{\perp}^{2} \sin ^{2} \theta_{0} \quad \Lambda_{2}=\frac{\lambda_{\Sigma}}{\Lambda_{1}}
$$


The target contractions are measured through a series of symmetric zigzag structures with various $\theta_{0}$ and $\lambda_{\perp}$ (Figure $\left.3 \mathrm{~b}, \mathrm{~d}\right)$ using the different systems presented in Figure $2 \mathrm{~b}$. They show a very good agreement with our minimal model, where the value of $\lambda_{\perp}$ was independently measured. In many systems, the active stretches $\left(\lambda_{\perp}, \lambda_{\|}\right)$are fixed. An important conclusion here for shape programming is that the homogenized principal stretches may now span the continuous range between $\lambda_{\perp}$ and $\lambda_{\|}$by adjusting the zigzag angle $\theta_{0}$. For example, in the case of inflated fabric, where actuation is uniaxial $\left(\lambda_{\|}=1\right)$, zigzag patterning brings bi-axial strains with a varying degree of biaxiality $\Lambda_{1} / \Lambda_{2}$. However we note from Equation (2) that the area contraction ratio $\Lambda_{1} \Lambda_{2}=\lambda_{\Sigma}$ is constant, and that the principal stretches lie between $\lambda_{\perp}$ and $\lambda_{\|}$.

We now examine the case of periodic asymmetric zigzag patterns, where assembled bands have alternating length $D_{\alpha}$ and $D_{\beta}$, defining the asymmetry parameter $\rho=D_{\beta} / D_{\alpha}$ (Figure 3c). When such non-symmetric zigzag patches are activated, a shear deformation is induced, with a theoretical angle $\gamma$ (see Supporting Information for derivation) following

$$
\tan \gamma=\frac{1-\rho}{1+\rho} \frac{\lambda_{\|}^{2}-\lambda_{\perp}^{2}}{\lambda_{\Sigma}} \frac{\sin 2 \theta_{0}}{2}
$$

This shearing angle $\gamma$ can be used in a tubular structure to induce chiral behavior, or rotation accompanying axial shortening (Figure 3e). Simple geometrical combination of Equation (1) predicts that the width and height variations $\left(H / H_{0}=\Lambda_{1} ; W / W_{0}=\Lambda_{2}\right)$ of the unit cell are identical to the symmetric case, explaining why both cylinders in Figure 3e have the same radius and height. Our quantitative experimental measurements (Figure 3c) agree well with these predictions. The components of the general homogenized metric tensor, expressed in the frame of reference of the 
bisectrix, are then deduced (see Supporting Information)

$$
\bar{G}=\left(\begin{array}{cc}
\Lambda_{1}^{2} & \lambda_{\Sigma} \tan \gamma \\
\lambda_{\Sigma} \tan \gamma & \Lambda_{2}^{2}\left(1+\tan ^{2} \gamma\right)
\end{array}\right)
$$

where $\Lambda_{1,2}$, are defined in Equation (2), but are no longer the principal stretches. We again find that the area changes by the same factor $\sqrt{\operatorname{det}(\bar{G})}=\lambda_{\Sigma}$ independently of the zigzag geometry. This is because each patch undergoes separately the same area change $\lambda_{\Sigma}$, which is therefore also imposed to the global area. On the other hand, the maximal stretch factor for the average metric $\bar{G}$ cannot exceed that of its components (and would only be reached if the same direction is maximally stretched by both patches, see Supporting Information for a detailed argument), so that the principal stretches also lie in the range between $\lambda_{\perp}$ and $\lambda_{\|}$. Metrics produced by a non-symmetric zigzag are therefore equivalent to those achievable by a rotated symmetric zigzag (but with another internal angle $\theta_{0}$, see Figure S9, Supporting Information). Changing the angle $\theta_{0}$ and the asymmetry $\rho=D_{\alpha} / D_{\beta}$, the degree of biaxiality of the principal stretches and their directions can be controlled, as in Figure 3c,e. However, zigzag asymmetry does not produce principal stretches outside of the interval between $\lambda_{\perp}$ and $\lambda_{\|}$which is already covered by symmetric constructions. This observation would also apply to more complex periodic combination of zigzag bands, because the arguments above are general. In the phase space of reachable strains (Figure 2a), any patterning of the director field of an active material with $\left(\lambda_{\|}, \lambda_{\perp}\right)$ can therefore at most provide the continuous family of active strains $\left(\Lambda_{I}, \Lambda_{I I}\right)$ lying on the $\operatorname{arc} \Lambda_{I} \Lambda_{I I}=\lambda_{\|} \lambda_{\perp}$ between the points $\left(\lambda_{\|}, \lambda_{\perp}\right)$ and $\left(\lambda_{\perp}, \lambda_{\|}\right)$. For example, texturing the director field of a baromorph with a series of angles $\left(0^{\circ} \leq \theta_{0} \leq 90^{\circ}\right)$, one single active deformation (orange square in Figure 2a) generates a continuous family of principal strains (orange solid line). In what follows, we focus for simplicity on symmetric zigzags, which already cover all reachable principal strains. 
We demonstrate on simple shapes how modulations of the geometry of the unit cell of the zigzag can be used for shape programming. We exploit the principle of Gaussian morphing, namely, induce controlled Gaussian curvature by prescribing spatial modulations of the metric tensor of the surface. These modulations are assumed to occur on a large enough length scale such that, locally, the active stretches are the microscale averages given by Equation (2), (3) and (4) for uniform zigzag. Shape programming is therefore obtained through another change of length scale (the "macroscale" in Figure 1). We focus on shapes corresponding to surfaces of revolution. Cylindrical shapes are obtained from a rectangular patch with cartesian coordinates $\left(x_{1}, x_{2}\right)$ glued along edges $x_{1}=0$ and $x_{1}=\mathcal{L}_{0}$, and we concentrate on the case where the coordinate lines are mapped to parallel and meridians in the deployed, inflated shape. These are then principal directions of stretch, and we are led to study cases where the zigzag interfaces are along these lines. By axisymmetry, stretches are constant along parallels (the image of axis $\left(x_{1}\right)$ ), and can vary only with coordinate $x_{2}$ (along the meridians). Following theorema egregium, the Gaussian curvature $K$ here can be computed in this particular case where $\Lambda_{1} \Lambda_{2}=\lambda_{\Sigma}$ as

$$
K=-\frac{1}{2 \lambda_{\Sigma}^{2}}\left(\Lambda_{1}^{2}\right)^{\prime \prime}
$$

where' ${ }^{\prime}$ stands for the derivative with respect to $x_{2} \cdot{ }^{[38]}$

In the absence of zigzags, we have from Equation (2) that either $\Lambda_{1} \equiv \lambda_{\|}$or $\Lambda_{1} \equiv \lambda_{\perp}$. In this case, the only achievable shapes are cylinders (of either smaller radius or smaller height). Zigzags open the possibility of obtaining a much larger class of shapes, including cones and surfaces with positive or negative Gaussian curvature. We start by programming shapes with zero Gaussian curvature $K=0$, therefore with $\Lambda_{1} \Lambda_{1}^{\prime}=C$ kept constant, according to Equation (5). Considering first the open straight ribbon with length $\mathcal{L}_{0}$, which when inflated transforms into an angular section $\varphi$ of a flat annulus. Indeed, each straight line along the ribbon acquires a length $\Lambda_{1} \mathcal{L}_{0}$ and 
a geodesic curvature $\kappa_{g}=\Lambda_{1}^{\prime} / \lambda_{\Sigma}$, therefore describing a portion of circle with an angular sector $\varphi=\Lambda_{1} \mathcal{L}_{0} \kappa_{g}=\mathcal{L}_{0} C / \lambda_{\Sigma}$ (which is indeed the same for all lines). Simple geometry shows that if one glues the edges of such an angular sector $\varphi$ of an annulus, a cone is obtained, with half angle $\alpha$ given by $\sin \alpha=\varphi /(2 \pi)$ or

$$
\sin \alpha=\frac{\mathcal{L}_{0}}{2 \pi} \frac{\Lambda_{1} \Lambda_{1}^{\prime}}{\lambda_{\Sigma}}
$$

For the sake of visual effect, we choose to use the maximum possible experimental range $\left(\Lambda_{1}=1\right.$ and $\Lambda_{1}=\lambda_{\perp}$ ) on each sides of a ribbon with width $\mathcal{H}_{0}$, entirely defining the stretch distribution $\Lambda_{1}\left(x_{2}\right)$ according to Equation (5). Substituting into Equation (2), the zigzag angle $\theta_{0}\left(x_{2}\right)$ can be computed, which leads to customized zigzag patterns (Figure S13; see Supporting Information for description of pattern design). Ribbons with the same channel geometry, but with various length $\mathcal{L}_{0}$ were head-tail sealed into cylinders, which were observed to morph into truncated conical shape (Figure 4a) when inflated. In Figure 4b, a family of cones with increasing $\alpha$ is obtained when increasing $\mathcal{L}_{0}$. Various conical shapes, down to a flat annulus $(\alpha=\pi / 2)$ are adopted. The outlines of these truncated cones from our theoretical prediction are plotted as dashed line, and match quantitatively experimental pictures (Figure 4b).

Axisymmetric shapes with Gaussian curvature can also be programmed. One road is to use Equation (6) that extends to the local tangent angle $\alpha\left(x_{2}\right)$ for a general axisymmetric surface obtained from a tubular patch with circumference $\mathcal{L}_{0}$. For shapes with constant Gaussian curvature $K$, we rather directly integrate Equation (5). A zigzag pattern using the maximum range allowed for our fabric $\left(\Lambda_{1}=\lambda_{\perp}\right.$ to 1$)$ for a ribbon with height $\mathcal{H}_{0}$ is then entirely defined by Equation (5). Figure 4c,d shows ribbons heat-sealed according to such patterns which are closed into cylinder and inflated. In our experiments with fabric, the maximal programmable Gaussian curvature reads $K=4\left|\lambda_{\perp}^{-2}-\lambda_{\|}^{-2}\right| / \mathcal{H}_{0}^{2}=4\left(\lambda_{\perp}^{-2}-1\right) / \mathcal{H}_{0}^{2}$. Different shapes are obtained for different cylinder perimeter $\mathcal{L}_{0}$. For positive curvature $K$, the specific case of a portion of a sphere is obtained for 
$\mathcal{L}_{0}=\mathcal{L}_{K}=2 \pi K^{-1 / 2}$, whereas other values lead to portions of spindles (which can be obtained by removing/adding and glueing an angular sector of a sphere along two meridians) in agreement with experiments. By programming homogeneous negative Gaussian curvature, cylinders can be turned into hyperboloid-like axisymmetric shapes for small value of $\mathcal{L}_{0} / \mathcal{L}_{K}$ (Figure $4 \mathrm{e}-\mathrm{h}$ ). For larger values, we observe a buckling instability breaking the rotational symmetry. This is probably due to the fact that bending stiffness of the fabric patterns with angles $\theta_{0}$ close to 0 or $\pi / 2$ is very anisotropic. The strong stiffness along the direction of the seams penalizes the curvature in the meridian plane that would increase with the perimeter. We also show in Supporting Material that non-constant Gaussian curvature may be programmed.

Beyond geometry, responsive materials along a director field often exhibit strong orthotropic mechanical properties (specifically for inflatable fabrics, ${ }^{[33]}$ and fibre reinforced hydrogels ${ }^{[21]}$ ). Such anisotropy may alter both the structural stiffness and the shape of the programmed shells, since current inverse problem methods are purely geometric and completely oversee the mechanics. Zigzag patterns appear thus as a way to get a more isotropic material response at the mesoscale, and thus a better structural stability of the shells. The precise homogenization of the mechanical properties (and more precisely, the bending stiffness) is beyond the scope of this article and will be the subject of future work. In order to illustrate this additional advantage of texturing, external loads are applied to two cylinders, one with a straight director and the other with a zigzag pattern. It clearly appears that the zigzag textures enables the latter cylinder to better resist external loads implying bending perpendicular to the director direction (Figure 5a). With a view towards applications, we also show in Figure 5b,c how this concept can be used to obtain stiff threedimensional shells objects very simply out of planar fabric.

In this article we have described a method to program the deployed shape of a sheet of material which is active along a vector field. We have considered materials for which the orientation of 
the vector field can be prescribed, but the magnitude of the active stretches is fixed. Using a zigzag construction, one may gain additional degrees of freedom for the actuation metric, namely the degree of biaxiality of the distortion (depending on the zigzag angle $\theta_{0}$ ) and the orientation of its principal axes (depending on the asymmetry ratio $\rho$ ). The method is based on a hierarchy of length scales: at a "microscopic" scale, the material undergoes a homogeneous stretch oriented along a specific material direction, with a fixed magnitude. These directions are combined into patches of zigzags obtained form the periodic repetition of a unit cell. The resulting deformations can be averaged at a "mesoscopic scale" (that of the zigzag patches) leading to a wider family of active stretches, that now depend on the geometry of the unit cell (parameters $\theta_{0}, \rho$ ). This wider palette of local active stretches can then be modulated at the larger scale of the structure, where their gradients are required to induce the Gaussian curvature necessary for the target shape. These long wavelength modulations can be obtained with slow variations of the unit cell of the zigzags. Sharp transitions in the zigzag angles would indeed induce reference metric discontinuities, leading to transition layers and involving the build-up of elastic stresses. ${ }^{[20,39]}$ We demonstrate the concept using two pneumatic systems (using inextensible fabric and stretchable elastomers) and 3D-printed thermoplastic shrinking along the printing direction above a critical temperature. We take the simple example of cylinders turned into cones and into axisymmetric shapes with positive and negative Gaussian curvature, for which we provide simple analytic formulas.

More theoretical work is needed to use the strategy to its full extent, as we have only focused on simple axisymmetric shapes, where the zigzag directing angle was constant along one direction (direction 2, the axis of the cylinder). In this study we have programmed the metric compatible with the desired 3D shells. But it would also be useful to program the (extrinsic) curvature directly in addition to the metric. Future work should determine to which extent the zigzag assembly could be used in this respect. Introducing cuts in the sheets could allow to further enlarge the parameter 
space of metric distortion, since it potentially relaxes the area contraction constraint highlighted in this study. We have shown here that the shape programming works surprisingly well, even if we did not use a large difference between scales in the experiments. Indeed the macroscale is hardly a few times larger than the microscale in patterns of Figure 4. The fact that this approach works so well even outside its intended domain of validity is encouraging, and it remains to be determined if this is specific for the axisymmetric case. We finally stress that the method is general, and envision that these results could be applied to other systems (such as liquid crystal elastomers), and at any scale.

\section{Experimental Section}

Inflatable fabrics: The fabrication process is completely 2D: we mount a soldering iron on a CNCmachine to directly print zigzag on two superimposed layers of inextensible sheets (thermoplasticurethane (TPU) coated nylon fabrics from Extremtextil) (Movie S1, Supporting Information). While printing at a given appropriate temperature, TPU matrix are welded together to obtain a strong bonding along the print path. ${ }^{[40,41]}$ Upon inflation, the sheets bend perpendicular to the parallel seam lines to form a quasi-circular cross section for sufficiently large pressure $p$. Owing to the quasi-inextensibility of the fabric sheets, this cross-section change implies an in-plane contraction perpendicular to the channels with a magnitude $\lambda_{\perp}=2 / \pi$ and $\lambda_{\|}=1$. Experimental measurement of $\lambda_{\perp}$ performed on patches with uniform parallel seams lead to $\lambda_{\perp}=0.73$ (thin fabric with a $70 \mathrm{~g} / \mathrm{qm}$ weight per unit surface) and $\lambda_{\perp}=0.77$ (thicker fabric with $170 \mathrm{~g} / \mathrm{qm}$ ), larger than the idealized $2 / \pi=0.63$ prediction. This is due to the non-zero size of the seam, and the finite bending stiffness of the fabric.

Baromorph: are obtained in a 2-steps process, using silicon elastomers that cure from a liquid mix (Zhermack Elite double, Young modulus $E=250 k P a$, see Supporting Information). ${ }^{[16]}$ Uncured 
elastomer is poured in 3D-printed moulds corresponding to the bottom part of the sample, which is later sealed by a uniform thickness layer of the same material. The total thickness of the plate was $5 \mathrm{~mm}$, with internal hollow channels with a rectangular section with height $3 \mathrm{~mm}$ and width in the range $1-2 \mathrm{~mm}$, to ensure a local in-plane channel density of $1 / 2$. Several pressure difference were imposed on uniform zigzag patterns, and the microscopic stretches $\left(\lambda_{\perp}, \lambda_{\|}\right)$were measured through image analysis.

$3 D$ printing: PolyLactic Acid (PLA filament, diameter of $1.75 \mathrm{~mm}$ ) is extruded at $200^{\circ} \mathrm{C}$ with a Fused Filament Fabrication 3D-printer (Hyrel 16A) and laid on a low temperature bed $\left(40^{\circ} \mathrm{C}\right)$ at relatively high speed $(4000 \mathrm{~mm} / \mathrm{min})$. When heated to $80^{\circ} \mathrm{C}$, such filament is observed to shrink axially by a factor $\lambda_{\|}$that depends mainly on the thickness of the deposited layer (see Supporting Information for more details).

In each system the global average metric was obtained through analyzing images of patches with uniform zigzag patterns, comparing at a large scale the rest and actuated states. Being uniform, such samples remain flat upon actuation.

\section{Supporting Information}

Supporting Information is available from the Wiley Online Library or from the author.

\section{Acknowledgements}

This work was supported by ESPCI Chaire Joliot-Curie, Chinese Scholarship Council Scholarship,

the French Agence Nationale de Recherche (ANR) for project SMART (ANR-15-CE08-0007) and the European Research Council (AdG-340685- MicroMotility).

\section{Conflict of Interest}

The authors declare no conflict of interest. 


\section{References}

[1] J. M. McCracken, B. R. Donovan, T. J. White, Advanced Materials 2020, 32, 201906564.

[2] K. Bhattacharya, R. D. James, Science 2005, 307, 570653.

[3] Q. Li, Intelligent stimuli-responsive materials: from well-defined nanostructures to applications, John Wiley \& Sons, 2013.

[4] S. Timoshenko, J. Optical Soc. Am 1925, 11233.

[5] R. F. Shepherd, F. Ilievski, W. Choi, S. A. Morin, A. A. Stokes, A. D. Mazzeo, X. Chen, M. Wang, G. M. Whitesides, Proceedings of the National Academy of Sciences 2011, 108, 51 20400.

[6] Y. Klein, E. Efrati, E. Sharon, Science 2007, 315.

[7] E. Efrati, E. Sharon, R. Kupferman, Journal of the Mechanics and Physics of Solids 2009, 57,4762 .

[8] J. Kim, J. A. Hanna, M. Byun, C. D. Santangelo, R. C. Hayward, Science 2012, 335, 6073 1201.

[9] E. Hajiesmaili, D. R. Clarke, Nature Communications 2019, 10, 1183.

[10] Y. Sawa, K. Urayama, T. Takigawa, A. DeSimone, L. Teresi, Macromolecules 2010, 43, 9 4362.

[11] T. H. Ware, M. E. McConney, J. J. Wie, V. P. Tondiglia, T. J. White, Science 2015, 347, 6225982.

[12] M. Warner, Annual Review of Condensed Matter Physics 2019, 11. 
[13] H. Aharoni, Y. Xia, X. Zhang, R. D. Kamien, S. Yang, Proceedings of the National Academy of Sciences 2018, 115, 287206.

[14] E. C. Davidson, A. Kotikian, S. Li, J. Aizenberg, J. A. Lewis, Advanced Materials 2020, 32, 11905682.

[15] A. S. Kuenstler, Y. Chen, P. Bui, H. Kim, A. DeSimone, L. Jin, R. C. Hayward, Advanced Materials 2020, 32, 172000609.

[16] E. Siéfert, E. Reyssat, J. Bico, B. Roman, Nature materials 2019, 18, 124.

[17] M. Arroyo, L. Heltai, D. Millán, A. DeSimone, Proceedings of the National Academy of Sciences 2012.

[18] Y.-L. Zhao, J. F. Stoddart, Langmuir 2009, 25, 158442.

[19] M. Yang, Z. Yuan, J. Liu, Z. Fang, L. Fang, D. Yu, Q. Li, Advanced Optical Materials 2019, 7, 161970059.

[20] Z. L. Wu, M. Moshe, J. Greener, H. Therien-Aubin, Z. Nie, E. Sharon, E. Kumacheva, Nature Communications 2013.

[21] R. M. Erb, J. S. Sander, R. Grisch, A. R. Studart, Nature Communications 2013, 4, 11.

[22] A. Sydney Gladman, E. A. Matsumoto, R. G. Nuzzo, L. Mahadevan, J. A. Lewis, Nature Materials 2016, 15, 413.

[23] J. W. Boley, W. M. van Rees, C. Lissandrello, M. N. Horenstein, R. L. Truby, A. Kotikian, J. A. Lewis, L. Mahadevan, Proceedings of the National Academy of Sciences 2019, 116, 42 20856.

[24] J. Gu, D. E. Breen, J. Hu, L. Zhu, Y. Tao, T. Van de Zande, G. Wang, Y. J. Zhang, L. Yao, 
In Proceedings of the 2019 CHI Conference on Human Factors in Computing Systems. 2019 $1-10$.

[25] I. Griniasty, H. Aharoni, E. Efrati, Physical review letters 2019, 123, 12127801.

[26] P. Plucinsky, M. Lemm, K. Bhattacharya, Physical Review E 2016, 94, 1010701.

[27] C. Kittel, Reviews of modern Physics 1949, 21, 4541.

[28] A. De Simone, Archive for rational mechanics and analysis 1993, 125, 299.

[29] M. S. Wechsler, D. Lieberman, T. Read, Trans. Aime 1953, $197,111503$.

[30] J. Bowles, J. Mackenzie, Acta metallurgica 1954, 2, $1129 ? 147$.

[31] K. Bhattacharya, A. DeSimone, K. Hane, R. D. James, C. Palmstrøm, Materials Science and Engineering: A 1999, 273685.

[32] M. Warner, E. M. Terentjev, Liquid crystal elastomers, volume 120, Oxford University Press, 2007.

[33] E. Siéfert, E. Reyssat, J. Bico, B. Roman, Soft Matter 2020.

[34] B. An, Y. Tao, J. Gu, T. Cheng, X. Chen, X. Zhang, W. Zhao, Y. Do, S. Takahashi, H.-Y. Wu, et al., In Proceedings of the 2018 CHI conference on human factors in computing systems. 2018 1-12.

[35] W. G. Yang, H. Lu, W. M. Huang, H. J. Qi, X. L. Wu, K. Y. Sun, Polymers 2014, 6, 82287.

[36] P. T. Mather, X. Luo, I. A. Rousseau, Annual Review of Materials Research 2009, 39445.

[37] E. Siéfert, M. Warner, Proceedings of the Royal Society A 2020.

[38] G. Cicconofri, M. Arroyo, G. Noselli, A. DeSimone, International Journal of Non-Linear Mechanics 2020, 118103278. 
[39] M. Moshe, E. Sharon, R. Kupferman, Nonlinearity 2013, 26, 123247.

[40] J. Ou, M. Skouras, N. Vlavianos, F. Heibeck, C.-Y. Cheng, J. Peters, H. Ishii, In Proceedings of the 29th Annual Symposium on User Interface Software and Technology. ACM, 2016 121-132.

[41] E. Siéfert, E. Reyssat, J. Bico, B. Roman, Proceedings of the National Academy of Sciences 2019, 116, 3416692 . 


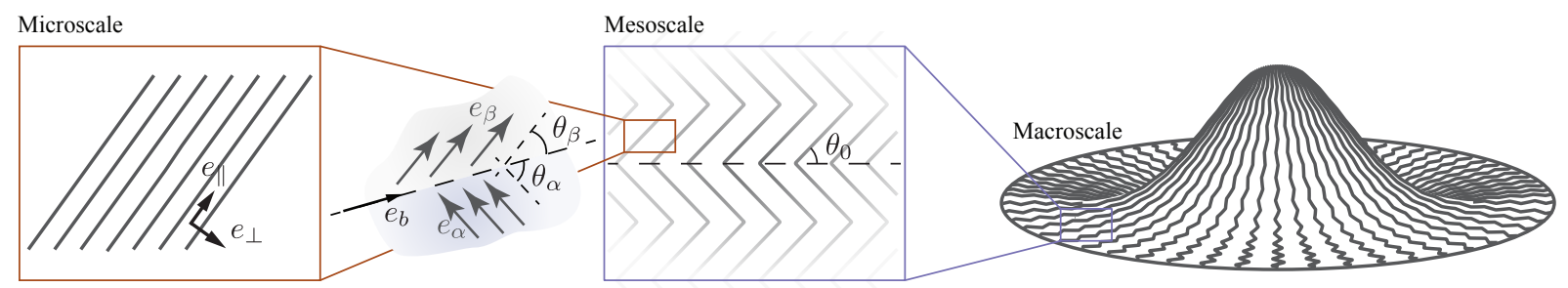

Figure 1: Zigzag texturing for Gaussian morphing. Taking advantage of a hierarchy of length scales, complex 3-dimensional shapes may be programmed. At the microscale, the material deforms along and perpendicular to a locally well-oriented director field (Equation (1)). Assembling patches of uniform directors requires the compatibility of deformation along the boundaries between patches; director fields $\mathbf{e}_{\alpha}, \mathbf{e}_{\beta}$ are oriented following a bisectrix rule (such that $\theta_{\alpha}=\theta_{\beta}$ denoted $\theta_{0}$ ). Zigzag patterns are thus obtained. Homogenizing the deformation of such patterns over a mesoscale, the local one-degree-of-freedom deformation of the material is transformed into an effective twodegree-of-freedom actuation. Varying the direction and the angle of the zigzags at a macroscale, 3-dimensional shapes may be programmed, exploiting the larger parameter space resulting from the zigzag texturing.

$\mathbf{a}$

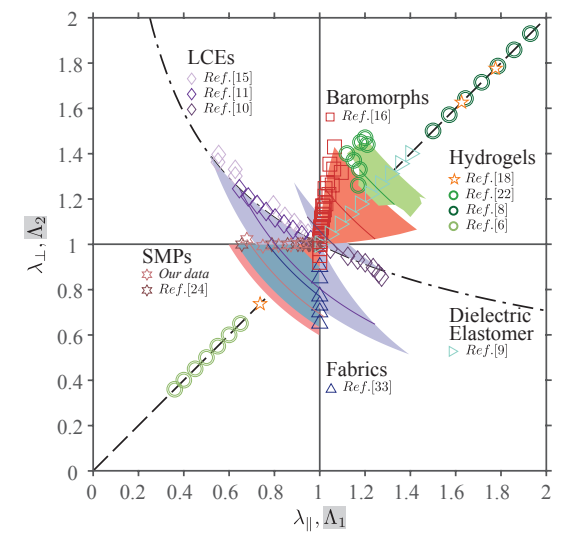

b



Elastomer

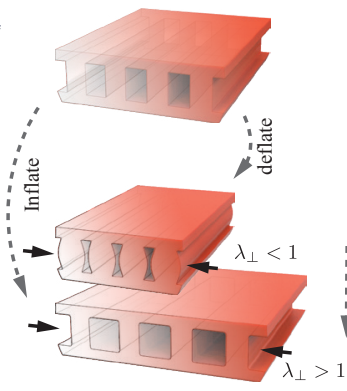

PLA

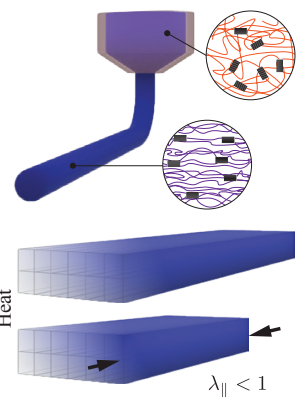

Figure 2: Active Materials (a) Phase space of principal actuation strains $\left(\lambda_{\|}, \lambda_{\perp}\right)$ for some state-of-the-art responsive materials (symbols). In all cases, the corresponding principal strains $\left(\Lambda_{1}, \Lambda_{2}\right)$ reachable with zigzag texturing cover a larger region (in color), except for conformal metric distortions $\left(\lambda_{\perp}=\lambda_{\|}\right.$, diagonal dashed line). Characteristic line for LCEs $\left(\lambda_{\perp}=\lambda_{\|}^{-0.5}\right)$ is also shown. (b) Actuation principle of the three responsive materials used during this study: (i) superimposed flat fabrics sheets welded together along locally parallel lines, inducing a contraction perpendicular to the seams direction upon inflation; (ii) channel bearing elastomers, which expand perpendicular to the channels direction when pressurized. (iii) 3D-printed PLA with a Fused Filament Fabrication (FFF) printer, which contracts along the printing direction when heated. 
a



b



c

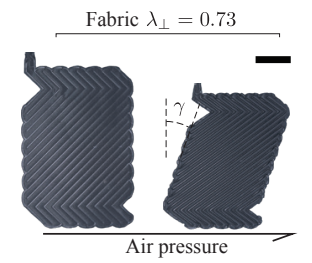

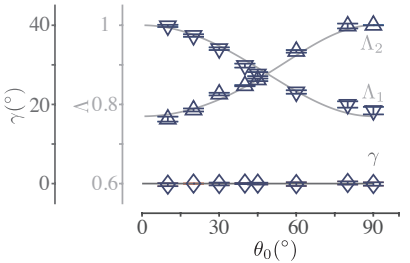

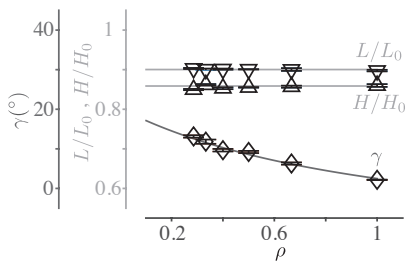

e

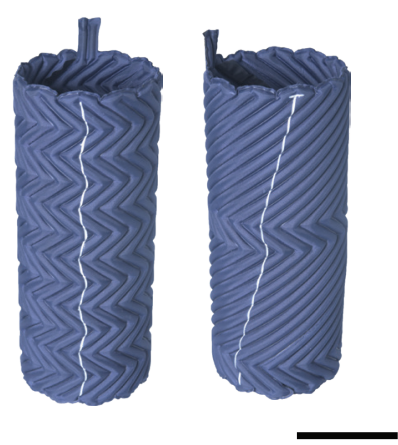

Figure 3: Homogenized deformation of zigzag unit cells. (a) Schematics of a symmetric (top) and asymmetric (bottom) unit cell in a reference (left) and deformed (right) states. (b-d) Experimental variation of width, height and shearing angle (right) for a zigzag unit cell with theory in solid line (experimental pictures on the left). (b) as a function of $\theta_{0}$ for $\rho=1, \lambda_{\perp}=0.77$ for a symmetric unit with inflatable fabrics (c) Asymmetric cell dimension reduction $W / W_{0}, H / H_{0}$ and shear angle $\gamma$ (bottom) as a function of $\rho\left(\theta_{0}=45^{\circ}, \lambda_{\perp}=0.73\right.$ ); (d) as a function of lateral expansion $\lambda_{\perp}$ with 3D-printed PLA filaments extruded at different thicknesses and pneumatic elastomers actuated with different pressure (experimental pictures on the left) (e) Inflated cylinders in the symmetric (right) and asymmetric (left) case: the white line is a straight, vertical line in the deflated state and exhibits the shear occurring in the asymmetric case. (Scale bars: $50 \mathrm{~mm}$ ) 
a

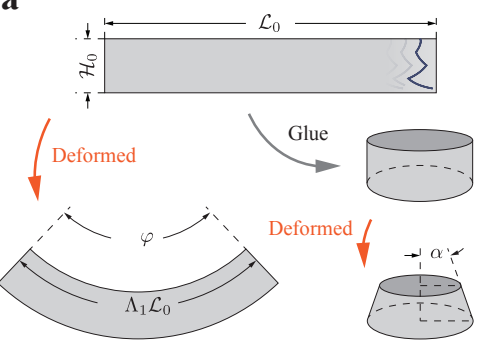

b

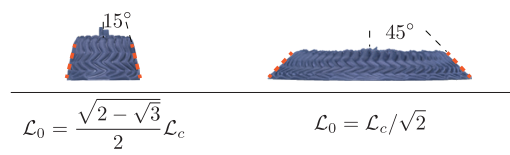

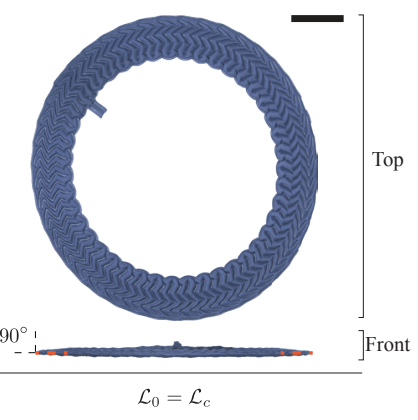

c

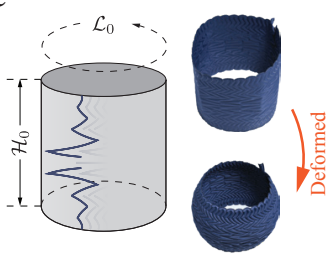

d


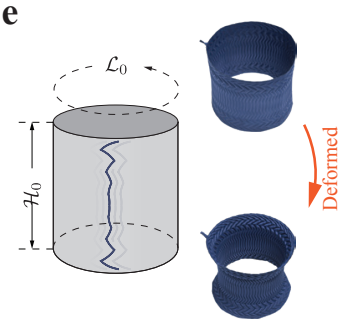

f

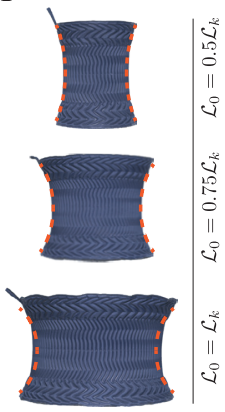

g

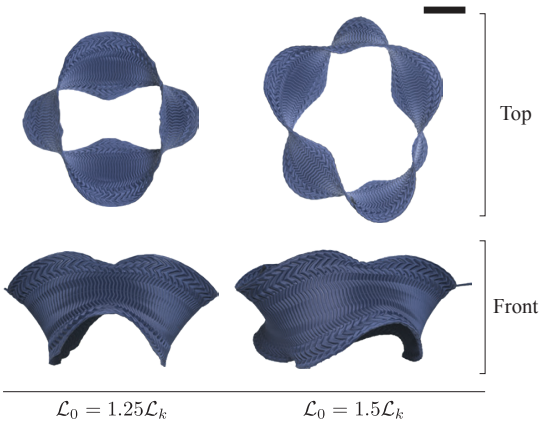

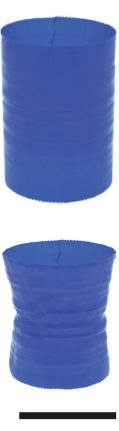

h

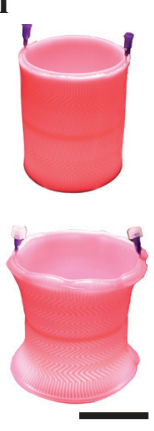

Figure 4: Programming simple axisymmetric shapes with zigzag patterns. (a) In-plane geodesic curvature (and thus zero Gaussian curvature) is programmed using Equation (5). When head-tail glued together, the ribbons of varying length form truncated cones with different angle $\alpha$ (b). Dashed red lines represent the theoretical prediction $\sin \alpha=\mathcal{L}_{0} / \mathcal{L}_{C}$, where $\mathcal{L}_{C}=4 \pi \mathcal{H}_{0} \lambda_{\perp} /\left[1-\lambda_{\perp}^{2}\right]\left(\lambda_{\perp}=0.77\right.$ for this particular fabric); (c) Axisymmetric shapes with constant positive Gaussian curvature $K=4\left(\lambda_{\perp}^{-2}-1\right) / \mathcal{H}_{0}^{2}$. (d) Pictures of the experimental shapes with constant curvature $K$ and equatorial perimeter $\mathcal{L}_{0}$. Here $\mathcal{L}_{K}=2 \pi / K^{-1 / 2}$ is the predicted perimeter leading to a portion of a sphere (dashed red lines: programmed shape). (e) Programming constant negative Gaussian curvature structures. $\mathcal{L}_{K}$ is the deflated perimeter of the cylinder for which both principal curvatures equal in the inflated state. (f) Pictures of the structures together with the theoretical shape (dashed red lines). When $\mathcal{L}_{0}>\mathcal{L}_{K}$, experimental shapes break axisymmetry (see the two examples on the right). We interpret this surprising instability as the result of stiffness anisotropy in the structure. (g,h) 3D-printed PLA structure and elastomer baromorph structure with a zigzag pattern deforming into a negatively curved shape when heated or pressurized. (Scale bars: $50 \mathrm{~mm}$ ) 
a

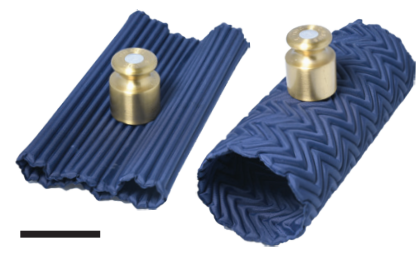

b

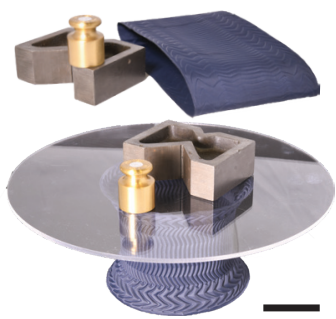

c

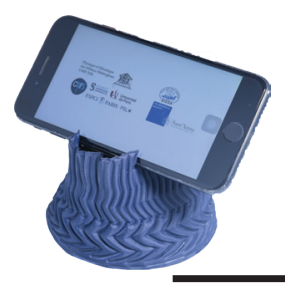

Figure 5: Structural stiffness of zigzag patterned shells (a) Zigzag textures enable the cylinder on the left to better resist external loads implying bending perpendicular to the director direction. (b) The inflated "hyperboloid table" (18 g) is stiff enough to support a circular plastic plate (316 g) and weights (1156g). (c) Phone (Pad) holder: Flat structures are easier to carry-on in the form of a roll for example and show their functionality when inflated. (Silvery Weight: 956 g; Golden Weight: 200 g; Scale bar: 50mm) 


\section{Table of Contents}

Many active materials used in shape-morphing respond to an external stimulus by stretching or contracting along a director field. Texturing this field in zigzag patterns is shown to provide an extended family of biaxial active stretches, opening a larger parameter space for shape control. This general notion of texturing over hierarchical length-scales can be applied to any anisotropically responsive material.

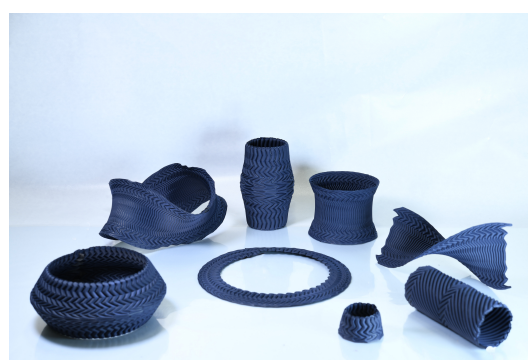

